\title{
THE EQUILIBRIUM RADIATIVE STATES OF A NONLINEAR QUANTUM-OPTICAL SYSTEM
}

\author{
N. N. Bogoliubov ${ }^{1}$, U. Taneri ${ }^{2}$, A. K. Prykarpatsky ${ }^{3}$ \\ ${ }^{1}$ Dept. of Statistical Mechanics at the Steklov Mathematical Institute of the Russian Academy of Sciences, \\ Moscow, 117966, Russian Federation \\ ${ }^{2}$ Eastern Mediterranean University of Famagusta, Department of Mathematics, N. Cyprus \\ ${ }^{3}$ University of Mining and Metallurgy of Kraków, Department of Applied Mathematics, Kraków 30059 Poland \\ and Eastern Mediterranean University of Famagusta, Department of Physics, N. Cyprus
}

(Received January 12, 2001; received in final form August 13, 2002)

\begin{abstract}
A lot of modern electronic devices are operating with optical signals generated due to the interaction of a working substance like crystal with an external laser radiation. As in most of these devices the interaction is nonlinear and processes making them stable are quantum, it is important to devise suitable approaches to studying their equilibrium radiative states subject to the external conditions imposed on a system. We succeded in finding a closed system of relationships on external crystal-light parameters yielding the quasi-equilibrium signal and idler intensity states radiated inside a crystal. This is the main aim of the presented work developing the modern quantum field theory methods for the analysis of radiative states in a nonlinear quantum-optical system having important applications in electronics.
\end{abstract}

Key words: equilibrium states, quantum optical models, Feynman diagrams, Green's functions.

PACS number(s): 71.30.Cc,78.40.Fy

\section{INTRODUCTION}

A great deal of studies was done concerning the problems of stability, bifurcation behaviour $[1,2]$ and dynamical properties [3-5] of atomic and molecular systems having applications in optical bi-stability of a laser cavity with a nonlinear crystal medium, in some microelectronics and other devices based on nonlinear interaction processes with radiation. Subject to multi-photon excitations of poly-atomic molecules undergoing also a selfinteraction via the Kerr effect the related processes can be modeled $[5,6]$ by means of the following quantumoptical approximated Hamiltonian operator

$$
\begin{aligned}
\hat{H} & =\hbar \omega_{0} a^{+} a-\hbar \chi_{0} a^{+} a^{2} a^{+} \\
& +\chi\left(a^{+} c+a c^{+}\right)+\hbar \omega_{1} c^{+} c,
\end{aligned}
$$

acting in a Fock space $\Phi$, where $\chi_{0} \in \mathbb{R}_{+}$and $\chi \in \mathbb{R}_{+}$ are coupling constant parameters, $a, a^{+}: \Phi \rightarrow \Phi$ are destruction and creation signal Bose-operators and $c, c^{+}$: $\Phi \rightarrow \Phi$ are these of an external radiation interacting with an active nonlinear media of a device considered.

If the coupling parameter $\chi=0$, then the nonperturbed Hamiltonian

$$
\hat{H}_{0}=\hbar \omega_{0} a^{+} a-\hbar \chi_{0} a^{+} a^{2} a^{+}+\hbar \omega_{1} c^{+} c
$$

is not quadratic in $\left(a, a^{+}\right)$-operator terms, but is such in the $\left(a^{+} a\right)$-terms:

$$
\hat{H}_{0}=\hbar\left(\omega_{0}-\chi_{0}\right) a^{+} a-\hbar \chi_{0}\left(a^{+} a\right)^{2}+\hbar \omega_{1} c^{+} c
$$

The latter obviously suggests a way for finding its spectrum in exact form based on that of the particle number operators $\hat{N}_{0}=a^{+} a, \hat{N}_{1}=c^{+} c$ :

$$
\hat{H}_{0}\left|\Omega_{m, n}\right\rangle:=E_{m, n}\left|\Omega_{m, n}\right\rangle
$$

where $\left|\Omega_{m, n}\right\rangle \in \Phi, m, n \in \mathbb{Z}_{+}$, via the standard Dirac brac-ket notation, and

$$
E_{m, n}=\hbar\left(\omega_{0}-\chi_{0}\right) m-\hbar \chi_{0} m^{2}+\hbar \omega_{1} n
$$

as $a^{+} a\left|\Omega_{m, n}\right\rangle=m\left|\Omega_{m, n}\right\rangle$ and $c^{+} c\left|\Omega_{m, n}\right\rangle=n\left|\Omega_{m, n}\right\rangle$ for all $m, n \in \mathbb{Z}_{+}$, can be easily constructed as follows:

$$
\left|\Omega_{m, n}\right\rangle:=\frac{\left(c^{+}\right)^{n}\left(a^{+}\right)^{m}}{\sqrt{m !} \sqrt{n !}}|0\rangle
$$

where $|0\rangle \in \Phi$ is the standard vacuum state of the Fock space $\Phi$. Thereby, we are now in a position to describe the ground state $|\Omega\rangle \in \Phi$ of our dynamical system (1.1) under nonzero interaction. Since the Hamiltonian operator describes a coherent absorption of an external radiation of frequency $\omega_{1}$ and formation of signal photon states of frequency $\omega_{0}$ the total radiative energy is not conserved, meaning thereby that the process described by the Hamiltonian (1.1) should be considered as dissipative.

Nevertheless, the task of finding the equilibrium ground state of the model Hamiltonian (1.1) is still in force owing to the non-conservation both of signal and 


\section{N. N. BOGOLIUBOV, U. TANERI, A. K. PRYKARPATSKY}

radiation photons generated by laser. Thus, we can consider our Hamiltonian (1.1) in the chemical potential expanded form

$$
\begin{aligned}
\hat{H}_{\mu} & =\hbar \omega_{0} a^{+} a-\chi_{0} a^{+} a^{2} a^{+}+\chi\left(a^{+} c+c^{+} a\right) \\
& +\hbar \omega_{1} c^{+} c-\mu_{0} a^{+} a-\mu_{1} c^{+} c
\end{aligned}
$$

where $\mu_{0}, \mu_{1} \in \mathbb{R}^{1}$ is a chemical potential of our system at the equilibrium ground state $\left|\Omega_{\mu}\right\rangle \in \Phi$. It can be determined from the following characteristic equilibrium conditions imposed on the system:

$$
\begin{aligned}
& \left.-\partial E_{\mu} / \partial \mu_{0}=<\Omega_{\mu}\left|a^{+} a\right| \Omega_{\mu}\right\rangle=N_{0}, \\
& \left.-\partial E_{\mu} / \partial \mu_{1}=<\Omega_{\mu}\left|c^{+} c\right| \Omega_{\mu}\right\rangle=N_{1}
\end{aligned}
$$

where $N_{0} \in \mathbb{Z}_{+}$is the equilibrium number of activated with signal photons molecular states in a crystal and $N_{1}$ $\in \mathbb{Z}_{+}$is the equilibrium number of external laser radiated photon states inside a crystal due to interaction. One can easily observe now from (1.8) that the total number

$$
N_{0}+N_{1}=\bar{N}_{1}
$$

is constant, being equal to a total number of radiated photons by laser. This equality is evidently true only in the case when the total amount $\bar{N}_{0}$ of active molecular states inside a crystal is greater than the amount $\bar{N}_{1}$ of photons radiated by laser. Anyway, the inequality $\bar{N}_{1} \leq \bar{N}_{0}$ is satisfieg in general by most of quantumoptical electronics devices. The condition (1.9) in the form

$$
-\left(\partial E_{\mu} / \partial \mu_{0}+\partial E_{\mu} / \partial \mu_{1}\right)=\bar{N}_{1}
$$

should be augmented still by another important physical condition

$$
\partial E_{\mu} / \partial N_{0}=0
$$

meaning evidently, that the ground state $\left|\Omega_{\mu}\right\rangle \in \Phi$ must be specified by the least energy $E_{\mu} \in \mathbb{R}$ subject to the amount of emitted signal photons $N_{0} \in \mathbb{Z}_{+}$owing to the interaction of a crystal with laser radiation. Two conditions (1.10) and (1.11) constitute obviously a complete set of constraints imposed on our ground state $\left|\Omega_{\mu}\right\rangle \in \Phi$ to be determined uniquely. For the above constraints to be implemented analytically we need to develop a technique for finding the quantities (1.8) in the functional form suitable for the analysis. This can be done based on the Feynman diagrammatic approach $[7,8]$ to interacting quantum fields.

\section{THE FEYNMAN DIAGRAM APPROACH: GREEN'S FUNCTIONS}

As is well known [7,8], the most effective method of finding the ground state characteristics of the Hamiltonian (1.1) under constraints (1.10) and (1.11) is making use of Feynman diagrammatic approach subject to the interaction Hamiltonian $\hat{H}_{\text {int }}=H_{\mu}-H_{0, \mu}$ in the interaction representation. Especially it proved to be of use for calculating the standard Green's functions [7] in the Heisenberg representation:

$$
\begin{aligned}
& i G_{0}\left(t, t^{\prime}\right):=\left\langle\Omega_{\mu}\right| T\left(a_{H_{\mu}}(t) a_{H_{\mu}}^{+}\left(t^{\prime}\right)\left|\Omega_{\mu}\right\rangle,\right. \\
& i G_{1}\left(t, t^{\prime}\right):=\left\langle\Omega_{\mu}\right| T\left(c_{H_{\mu}}(t) c_{H_{\mu}}^{+}\left(t^{\prime}\right)\left|\Omega_{\mu}\right\rangle\right.
\end{aligned}
$$

and the so-called anomalous Green's function

$$
i G_{01}\left(t, t^{\prime}\right):=\left\langle\Omega_{\mu}\right| T\left(c_{H_{\mu}}(t) a_{H_{\mu}}^{+}\left(t^{\prime}\right)\left|\Omega_{\mu}\right\rangle,\right.
$$

where the operation " $T$ " denotes the standard chronological operator ordering, giving rise to the following expression for the ground state energy $E_{\mu} \in \mathbb{R}$ :

$$
\begin{aligned}
E_{\mu}: & =\left\langle\Omega_{\mu}\left|\hat{H}_{\mu}\right| \Omega_{\mu}\right\rangle=\left\langle\Omega_{\mu}\left|\hat{H}_{0, \mu}\right| \Omega_{\mu}\right\rangle \\
& -\left.i \chi\left(G_{01}\left(t, t^{+}\right)+G_{01}^{*}\left(t, t^{+}\right)\right)\right|_{t=0} .
\end{aligned}
$$

Owing to the following simply verified general expression

$$
E_{\mu}=E_{0, \mu}+\int_{0}^{1} d \ln \lambda\left\langle\Omega_{\mu}(\lambda)\left|\lambda \hat{H}_{i n t}\right| \Omega_{\mu}(\lambda)\right\rangle
$$

where $\left|\Omega_{\mu}(\lambda)\right\rangle \in \Phi$ is the ground state corresponding to the scaled interaction Hamiltonian $\lambda \hat{H}_{\text {int }}: \Phi \rightarrow \Phi$, and $E_{0, \mu} \in \mathbb{R}$ is the ground state energy of the nonperturbed Hamiltonian $\hat{H}_{0, \mu}: \Phi \rightarrow \Phi$, one can determine (2.3) only due to the anomalous Green's function (2.2) redefined as

$$
i G_{01, \lambda}\left(t, t^{\prime}\right)=\left\langle\Omega_{\mu}(\lambda)\right| T\left(c_{H_{\mu}(\lambda)}(t) a_{H_{\mu}(\lambda)}^{+}\left(t^{\prime}\right)\left|\Omega_{\mu}(\lambda)\right\rangle,\right.
$$

where $\lambda \in(0,1]$ is a scaling parameter. Namely, from (2.4) and (2.5) it follows that

$$
E_{\mu}=E_{0, \mu}-\left.i \chi \int_{0}^{1} d \lambda\left(G_{01, \lambda}\left(t, t^{+}\right)+G_{01, \lambda}^{*}\left(t, t^{+}\right)\right)\right|_{t=0}
$$


where the energy $E_{0, \mu}$ is found easily owing to the expression (1.6):

$$
E_{0, \mu}=\hbar\left(\omega_{0}-\chi_{0}-\mu_{0} / \hbar\right) N_{0}-\hbar \chi_{0} N_{0}^{2}+\hbar \omega_{1}\left(N_{1}-\mu_{1} / \hbar\right) .
$$

Whence we arrived at the need to calculate just the anomalous Green's function (2.5) subject to the scaled interaction Hamiltonian $\lambda \hat{H}_{\mathrm{int}}: \Phi \rightarrow \Phi$ for all $\lambda \in(0,1]$. This problem we shall discuss in detail below.

\section{THE FEYNMAN DIAGRAM APPROACH: PERTURBATION SERIES EXPANSION}

Subject to the Green's functions (2.1) and (2.2) we need to define the nonperturbed ground state $\left|\Omega_{0, \mu}\right\rangle \in \Phi$, satisfying the equation

$$
\hat{H}_{0, \mu}\left|\Omega_{0, \mu}\right\rangle=E_{0, \mu}\left|\Omega_{0, \mu}\right\rangle,
$$

where $E_{0, \mu}$ is given by the expression (2.7). Owing to the fact that photons are Bose-particles, we are forced to make shifts in $\left(a, a^{+}\right)$and $\left(c, c^{+}\right)$-operators producing so called Bose condensates [9] subject to constraints $(1.8)$ :

$$
\begin{aligned}
& a: \Longrightarrow \tilde{a}+\sqrt{N_{0}}, \quad a^{+}: \Longrightarrow \tilde{a}^{+}+\sqrt{N_{0}}, \\
& c: \Longrightarrow \tilde{c}+\sqrt{N_{1}}, \quad c^{+}: \Longrightarrow \tilde{c}^{+}+\sqrt{N_{1}},
\end{aligned}
$$

where operators $\tilde{a}$ and $\tilde{c}$ satisfy the following no-particle vacuum state conditions:

$$
\tilde{a}\left|\Omega_{0, \mu}\right\rangle=0, \quad \tilde{c}\left|\Omega_{0, \mu}\right\rangle=0 .
$$

Subject to transformations (3.2) our interaction Hamiltonian $\hat{H}_{\text {int }}: \Phi \rightarrow \Phi$ becomes like

$$
\begin{aligned}
\hat{H}_{i n t} & =\chi\left[\left(\tilde{a}^{+}+\sqrt{N_{0}}\right)\left(\tilde{c}+\sqrt{N_{1}}\right)\right. \\
& \left.+\left(\tilde{a}+\sqrt{N_{0}}\right)\left(\tilde{c}^{+}+\sqrt{N_{1}}\right)\right] \\
& =\chi\left[\left(\tilde{a}^{+} \tilde{c}+\tilde{a} \tilde{c}^{+}\right)+\left(\tilde{c} \sqrt{N_{0}}+\tilde{a} \sqrt{N_{1}}\right)\right. \\
& \left.\left.+\left(\tilde{c}^{+} \sqrt{N_{0}}+\tilde{a}^{+} \sqrt{N_{1}}\right)\right]+2 \chi \sqrt{N_{0} N_{1}}\right] .
\end{aligned}
$$

This means that the nonperturbed Hamiltonian $\hat{H}_{0, \mu}$ should be modified by the constant term $2 \chi \sqrt{N_{0} N_{1}}$ giving rise to the expression:

$$
\tilde{H}_{0, \mu}=\hbar\left(\omega_{0}-\mu_{0} / \hbar\right)\left(\tilde{a}^{+}+\sqrt{N_{0}}\right)
$$

$$
\begin{aligned}
& \times\left(\tilde{a}+\sqrt{N_{0}}\right)+\hbar\left(\omega_{1}-\mu_{1} / \hbar\right) \\
& \times\left(\tilde{c}^{+}+\sqrt{N_{1}}\right)\left(\tilde{c}+\sqrt{N_{1}}\right)+\chi_{0}\left(\tilde{a}^{+}+\sqrt{N_{0}}\right) \\
& \times\left(\tilde{a}+\sqrt{N_{0}}\right)^{2}\left(\tilde{a}^{+}+\sqrt{N_{0}}\right)+2 \chi \sqrt{N_{0} N_{1}}
\end{aligned}
$$

Thus, the scaled modified interaction Hamiltonian becomes like

$$
\begin{aligned}
\lambda \tilde{H}_{\mathrm{int}} & =\lambda \chi\left[\left(\begin{array}{lll}
\tilde{a}^{+} & \tilde{c}+\tilde{a} & \tilde{c}^{+}
\end{array}\right)\right. \\
& \left.+\left(\tilde{c}+\tilde{c}^{+}\right) \sqrt{N_{0}}+\left(\tilde{a}^{+}+\tilde{a}\right) \sqrt{N_{1}}\right]
\end{aligned}
$$

where $\lambda \in(0,1]$.

Consider now the modified ground state $\left|\tilde{\Omega}_{\mu}\right\rangle \in \Phi$, satisfying the equation like (3.1):

$$
\tilde{H}_{0, \mu}\left|\tilde{\Omega}_{0, \mu}\right\rangle=\tilde{E}_{0, \mu}\left|\tilde{\Omega}_{0, \mu}\right\rangle
$$

Since transformation (3.2) is linear the spectrum of operator (3.5) coincides with that of the operator $\hat{H}_{0, \mu}$ up to the constant shift $2 \chi \sqrt{N_{0} N_{1}}$. Thus,

$$
\begin{aligned}
\tilde{E}_{0, \mu} & =\left(\hbar \omega_{0}-\chi_{0}-\mu_{0} / \hbar\right) N_{0}-\hbar \chi_{0} N_{0}^{2} \\
& +\hbar \omega_{1}\left(N_{1}-\mu_{1} / \hbar\right)+2 \chi \sqrt{N_{0} N_{1}}
\end{aligned}
$$

and we arrived at the starting point of formula (2.4):

$$
\tilde{E}_{\mu}=\tilde{E}_{0, \mu}+\int_{0}^{1} d \ln \lambda\left\langle\tilde{\Omega}_{\mu}(\lambda)\left|\lambda \tilde{H}_{i n t}\right| \tilde{\Omega}_{\mu}(\lambda)\right\rangle
$$

where the term $\lambda \tilde{H}_{\text {int }}$ is given by (3.6). For the matrix element in (3.9) to be calculated in an analytical form, it is necessary to find all Green's functions modifying (2.1) and (2.2):

$i \tilde{G}_{0, \lambda}\left(t, t^{\prime}\right):=\left\langle\tilde{\Omega}_{\mu}(\lambda)\left|T\left(\tilde{a}_{\tilde{H}_{\mu}}(t) \tilde{a}_{\tilde{H}_{\mu}}^{+}\left(t^{\prime}\right)\right)\right| \tilde{\Omega}_{\mu}(\lambda)\right\rangle$,

$i \tilde{G}_{0, \lambda}\left(t, t^{\prime}\right):=\left\langle\tilde{\Omega}_{\mu}(\lambda)\left|T\left(\tilde{c}_{\tilde{H}_{\mu}}(t) \tilde{c}_{\tilde{H}_{\mu}}^{+}\left(t^{\prime}\right)\right)\right| \tilde{\Omega}_{\mu}(\lambda)\right\rangle$,

and

$i \tilde{G}_{01, \lambda}\left(t, t^{\prime}\right):=\left\langle\tilde{\Omega}_{\mu}(\lambda)\left|T\left(\tilde{a}^{+}\left(t^{\prime}\right) \tilde{c}(t)\right)\right| \tilde{\Omega}_{\mu}(\lambda)\right\rangle$. 
Owing to general Feynman rules of finding Green's functions (3.10) and (3.11) it is necessary first to calculate all nonperturbed Green's functions at $\chi=0$, that is functions

$$
\begin{aligned}
& i \tilde{G}_{0}^{(0)}\left(t, t^{\prime}\right):=\left\langle\tilde{\Omega}_{0, \mu}\left|T\left(\tilde{a}_{\tilde{H}_{0, \mu}}(t) \tilde{a}_{\tilde{H}_{0, \mu}}^{+}\left(t^{\prime}\right)\right)\right| \tilde{\Omega}_{0, \mu}\right\rangle \\
& i \tilde{G}_{0}^{(0)}\left(t, t^{\prime}\right):=\left\langle\tilde{\Omega}_{0, \mu}\left|T\left(\tilde{c}_{\tilde{H}_{0, \mu}}(t) \tilde{c}_{\tilde{H}_{0, \mu}}^{+}\left(t^{\prime}\right)\right)\right| \tilde{\Omega}_{0, \mu}\right\rangle
\end{aligned}
$$

for all times $t, t^{\prime} \in \mathbb{R}^{1}$. Due to the result (3.8) the Green's functions can be found explicitly after some simple but a bit cumbersome calculations as follows: for photon creation-annihilation Green's function one gets

$$
\begin{aligned}
\tilde{G}_{0}^{(0)}\left(t, t^{\prime}\right) & =N_{0} \exp \left[i\left(t-t^{\prime}\right)\left(\omega_{0}-\chi_{0}-\mu_{0} / \hbar-2 \chi_{0} N_{0} / \hbar\right)\right]+\vartheta\left(t-t^{\prime}\right)+N_{0}\left\{\exp \left[i t\left(\omega_{0}-\chi_{0}-\mu_{0} / \hbar-2 \chi_{0} N_{0} / \hbar\right)\right]-1\right\} \\
& \times\left\{\exp \left[-i t^{\prime}\left(\omega_{0}-\chi_{0}-\mu_{0} / \hbar-2 \chi_{0} N_{0} / \hbar\right)\right]-1\right\} \\
\tilde{G}_{1}^{(0)}\left(t, t^{\prime}\right) & =N_{1} \exp \left[i\left(t-t^{\prime}\right)\left(\omega_{1}-\mu_{1} / \hbar\right)\right] \vartheta\left(t-t^{\prime}\right)+N_{1}\left\{\exp \left[i t\left(\omega_{1}-\mu_{1} / \hbar\right)\right]-1\right\}\left\{\exp \left[-i t^{\prime}\left(\omega_{1}-\mu_{1} / \hbar\right)\right]-1\right\}
\end{aligned}
$$

and for the anomalous Green's function one gets

$$
\tilde{G}_{01}^{(0)}\left(t, t^{\prime}\right)=0
$$

Thereby, we can express now Green's functions (2.1) via Feynman diagram series making use of the Dyson equations [7]:

$$
\begin{aligned}
& \tilde{G}_{0, \lambda}\left(t, t^{\prime}\right)=\tilde{G}_{0}^{(0)}\left(t, t^{\prime}\right)+\int_{\mathbb{R}} d \tau^{\prime} \int_{\mathbb{R}} d \tau^{\prime \prime} \tilde{G}_{0}^{(0)}(t, \tau) \widetilde{\sum_{0, \lambda}^{*}}\left(\tau, \tau^{\prime}\right) \tilde{G}_{0, \lambda}\left(\tau^{\prime}, t^{\prime}\right), \\
& \tilde{G}_{1, \lambda}\left(t, t^{\prime}\right)=\tilde{G}_{1}^{(0)}\left(t, t^{\prime}\right)+\int_{\mathbb{R}} d \tau^{\prime} \int_{\mathbb{R}} d \tau^{\prime \prime} \tilde{G}_{0}^{(0)}(t, \tau) \widetilde{\sum_{1, \lambda}^{*}}\left(\tau, \tau^{\prime}\right) \tilde{G}_{1, \lambda}\left(\tau^{\prime}, t^{\prime}\right),
\end{aligned}
$$

with kernels $\widetilde{\Sigma}_{0, \lambda}^{*}$ and $\widetilde{\Sigma}_{1, \lambda}^{*}$ called proper self-energy insertions (or mass operators) [7] and expressed in the following diagram form:

$$
\begin{aligned}
& -i \widehat{\sum_{0, \lambda}^{*}}\left(\tau, \tau^{\prime}\right)=
\end{aligned}
$$

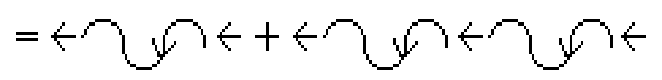

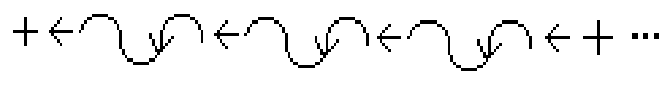

$$
\begin{aligned}
& =\left(\frac{i \lambda \chi}{\hbar}\right)^{2} \tilde{G}_{1}^{(0)}\left(\tau, \tau^{\prime}\right)+\left(\frac{i \lambda \chi}{\hbar}\right)^{4} \tilde{G}_{1}^{(0)} * \tilde{G}_{0}^{(0)} * \tilde{G}_{1}^{(0)}\left(\tau, \tau^{\prime}\right)+\ldots \\
& -i{\overline{\sum_{1, \lambda}^{*}}}^{*}\left(\tau, \tau^{\prime}\right)=
\end{aligned}
$$

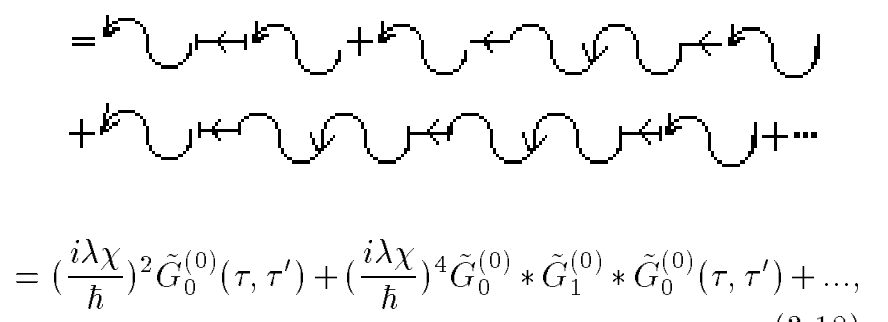

where we denoted by "*" the convolution operation between Green's functions, and

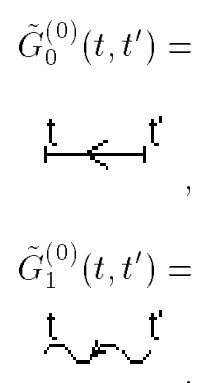

Thereby, we can now use of the constraints (1.10) and (1.11) for determining the chemical potentials $\mu_{0}$ and $\mu_{1}$. 
Based on expressions (3.17) and (3.18) one arrives at the following integral relationships for Green's functions (3.10):

$$
\begin{aligned}
& \tilde{G}_{0, \lambda}\left(t, t^{\prime}\right)=\tilde{G}_{0}^{(0)}\left(t, t^{\prime}\right)+\left(\frac{\lambda \chi}{\hbar}\right)^{2} \tilde{G}_{0}^{(0)} * \tilde{\mathcal{R}}_{0, \lambda}^{*}\left(t, t^{\prime}\right) \\
& \tilde{\mathcal{R}}_{0, \lambda}^{*}=\left(\frac{\lambda \chi}{\hbar}\right)^{2} \tilde{G}_{1}^{(0)} * \tilde{G}_{0}^{(0)} \\
& +\left(\frac{\lambda \chi}{\hbar}\right)^{2} \tilde{G}_{1}^{(0)} * \tilde{G}_{0}^{(0)} * \tilde{\mathcal{R}}_{0, \lambda}^{*} \\
& \tilde{G}_{1, \lambda}\left(t, t^{\prime}\right)=\tilde{G}_{1}^{(0)}\left(t, t^{\prime}\right)+\left(\frac{\lambda \chi}{\hbar}\right)^{2} \tilde{G}_{1}^{(0)} * \tilde{\mathcal{R}}_{1, \lambda}^{*}\left(t, t^{\prime}\right) \\
& \tilde{\mathcal{R}}_{1, \lambda}^{*}=\left(\frac{\lambda \chi}{\hbar}\right)^{2} \tilde{G}_{0}^{(0)} * \tilde{G}_{1}^{(0)} \\
& +\left(\frac{\lambda \chi}{\hbar}\right)^{2} \tilde{G}_{0}^{(0)} * \tilde{G}_{1}^{(0)} * \tilde{\mathcal{R}}_{1, \lambda}^{*} .
\end{aligned}
$$

An expression similar to the above-given ones can be written also for the Green's function (3.13) on what we shall not dwell here.

\section{THE EQUILIBRIUM STATE ANALYSIS}

As was mentioned before, for the equilibrium state $\left|\tilde{\Omega}_{\mu}\right\rangle \in \Phi$ to be specified completely, we need to implement constraints (1.10) and (1.11) on the energy value $\tilde{E}_{\mu}$ subject to chemical potentials $\mu_{0}$ and $\mu_{1}$. Since the nonperturbed part $\tilde{E}_{0, \mu}$ (3.8) of this energy is already determined, we need only calculate the integral part of $(3.9)$ :

$$
\begin{aligned}
\Delta \tilde{E}_{\mu} & =\int_{0}^{1} d \ln \lambda\left\langle\tilde{\Omega}_{\mu}(\lambda)\left|\lambda \tilde{H}_{i n t}\right| \tilde{\Omega}_{\mu}(\lambda)\right\rangle \\
& =\chi \int_{0}^{1} d \lambda\left\langle\tilde{\Omega}_{\mu}(\lambda)\right|\left[\tilde{a}^{+} \tilde{c}+\tilde{a} \tilde{c}^{+}\right) \\
& \left.+\sqrt{N_{1}}\left(\tilde{a}^{+}+\tilde{a}\right)+\sqrt{N_{0}}\left(\tilde{c}^{+}+\tilde{c}\right)\right] \mid \tilde{\Omega}_{\mu}(\lambda) \\
& =i \chi \int_{0}^{1} d \lambda\left[\tilde{G}_{01, \lambda}\left(0,0^{+}\right)+\tilde{G}_{01, \lambda}^{*}\left(0,0^{+}\right)\right] \\
& +\chi \sqrt{N_{1}} \int_{0}^{1} d \lambda\left[\tilde{A}_{\lambda}^{*}(0)+\tilde{A}_{\lambda}(0)\right] \\
& +\chi \sqrt{N_{0}} \int_{0}^{1} d \lambda\left[\tilde{C}_{\lambda}^{*}(0)+\tilde{C}_{\lambda}(0)\right],
\end{aligned}
$$

where we defined for any $t \in \mathbb{R}^{1}$

$$
\begin{aligned}
& \tilde{A}_{\lambda}(t):=\left\langle\tilde{\Omega}_{\mu}(\lambda)\left|\tilde{a}_{\tilde{H}_{\mu}(\lambda)}^{+}(t)\right| \tilde{\Omega}_{\mu}(\lambda)\right\rangle, \\
& \tilde{C}_{\lambda}(t):=\left\langle\tilde{\Omega}_{\mu}(\lambda)\left|\tilde{c}_{\tilde{H}_{\mu}(\lambda)}^{+}(t)\right| \tilde{\Omega}_{\mu}(\lambda)\right\rangle .
\end{aligned}
$$

The anomalous functions (4.2) can be easily calculated making use of the Feynman diagram expansions:

$$
\tilde{A}_{\lambda}(t)=
$$

$$
\begin{aligned}
& =\leftarrow^{\mathrm{t}}+\mathrm{g},
\end{aligned}
$$

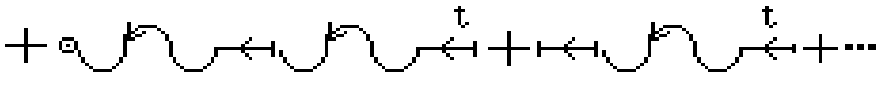

$$
\begin{aligned}
& \Longrightarrow \sqrt{N_{1}}\left(\frac{i \lambda \chi}{\hbar}\right) \tilde{G}_{0}^{(0)}(\cdot, t)+\sqrt{N_{1}}\left(\frac{i \lambda \chi}{\hbar}\right)^{3} \tilde{G}_{0}^{(0)}(\cdot) * \\
& * \tilde{G}_{1}^{(0)} * \tilde{G}_{0}^{(0)}(\cdot, t)+\ldots+\sqrt{N_{0}}\left(\frac{i \lambda \chi}{\hbar}\right)^{2} \tilde{G}_{1}^{(0)}(\cdot) * \tilde{G}_{0}^{(0)}(\cdot, t) \\
& +\sqrt{N_{0}}\left(\frac{i \lambda \chi}{\hbar}\right)^{4} \tilde{G}_{1}^{(0)}(\cdot) * \tilde{G}_{0}^{(0)} * \tilde{G}_{1}^{(0)} * \tilde{G}_{0}^{(0)}(\cdot, t)+\ldots \\
& =\tilde{A}_{0, \lambda}(t)+\tilde{A}_{1, \lambda}(t), \\
& \tilde{C}_{\lambda}(t)=
\end{aligned}
$$

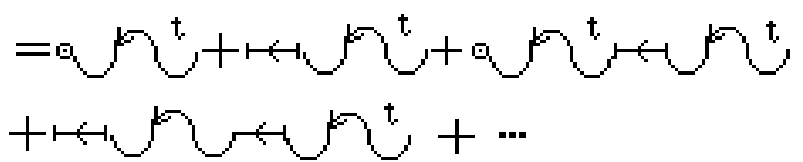

$$
\begin{gathered}
\Longrightarrow \sqrt{N_{0}}\left(\frac{i \lambda \chi}{\hbar}\right) \tilde{G}_{1}^{(0)}(\cdot, t)+\sqrt{N_{1}}\left(\frac{i \lambda \chi}{\hbar}\right)^{2} \tilde{G}_{0}^{(0)}(\cdot) * \tilde{G}_{1}^{(0)}(\cdot, t)+\ldots \\
+\sqrt{N_{0}}\left(\frac{i \lambda \chi}{\hbar}\right)^{3} \tilde{G}_{1}^{(0)}(\cdot) * \tilde{G}_{0}^{(0)} * \tilde{G}_{1}^{(0)}(\cdot, t)+\ldots \\
=\tilde{C}_{0, \lambda}(t)+\tilde{C}_{1, \lambda}(t)
\end{gathered}
$$

where by definition, the sign " $\odot$ " means a factor $\sqrt{N_{0}}$, the sign " $x$ " means a factor $\sqrt{N_{1}}$, an internal dot "." corresponds to a factor $(i \chi / \hbar)$, with the following Dyson's type relationships having to be satisfied:

$$
\begin{aligned}
\tilde{A}_{0, \lambda}(t) & =\sqrt{N_{1}}\left(\frac{i \lambda \chi}{\hbar}\right) \tilde{G}_{0}^{(0)}(\cdot, t) \\
& +\left(\frac{i \lambda \chi}{\hbar}\right)^{2} \tilde{A}_{0, \lambda}(t) * \tilde{G}_{1}^{(0)} * \tilde{G}_{0}^{(0)}(\cdot, t),
\end{aligned}
$$




$$
\begin{aligned}
\tilde{A}_{1, \lambda}(t) & =\sqrt{N_{0}}\left(\frac{i \lambda \chi}{\hbar}\right)^{2} \tilde{G}_{1}^{(0)}(\cdot) * \tilde{G}_{0}^{(0)}(\cdot, t) \\
& +\left(\frac{i \lambda \chi}{\hbar}\right)^{2} \tilde{A}_{1, \lambda}(t) * \tilde{G}_{1}^{(0)} * \tilde{G}_{0}^{(0)}(\cdot, t) \\
\tilde{C}_{0, \lambda}(t) & =\sqrt{N_{1}}\left(\frac{i \lambda \chi}{\hbar}\right)^{2} \tilde{G}_{0}^{(0)}(\cdot) * \tilde{G}_{1}^{(0)}(\cdot, t) \\
& +\left(\frac{i \lambda \chi}{\hbar}\right)^{2} \tilde{C}_{0, \lambda} * \tilde{G}_{0}^{(0)} * \tilde{G}_{1}^{(0)}(\cdot, t), \\
\tilde{C}_{1, \lambda}(t) & =\sqrt{N_{0}}\left(\frac{i \lambda \chi}{\hbar}\right) \tilde{G}_{1}^{(0)}(\cdot, t) \\
& +\left(\frac{i \lambda \chi}{\hbar}\right)^{2} \tilde{C}_{1, \lambda} * \tilde{G}_{0}^{(0)} * \tilde{G}_{1}^{(0)}(\cdot, t) .
\end{aligned}
$$

Having solved equations (4.4) and (4.5) we arrive due to expressions (4.3) and (4.1) at the energy shift $\Delta \tilde{E}_{\mu}$ which makes it possible to satisfy constraints (1.10) and (1.11), where $E_{\mu}$ should be replaced by $\tilde{E}_{\mu}$ :

$$
E_{\mu}: \Longrightarrow \tilde{E}_{\mu}=\tilde{E}_{0, \mu}+\Delta \tilde{E}_{\mu}
$$

and $\tilde{E}_{0, \mu}$ is given by expression (3.8). In the case when the susceptibility parameter $\chi \in \mathbb{R}_{+}$is too small, equations (4.4) and (4.5) can be easily solved, where by making it possible to implement the constraints (1.10) and (1.11) into the analysis of the ground state energy $E_{\mu}$ $\in \mathbb{R}$.

Thereby, we succeded in finding a closed system of relationships on light and crystal parameters giving rise to the signal and idler intensities of radiated states inside a crystal. An analysis of this system relative to nonequilibrium dynamical properities of the model will be a subject of next Part 2 of this work under preparation.

\section{ACKNOWLEDGEMENTS}

The authors are grateful to Profs. M. Halilsoy and E. Aydiroglu for interesting discussions. One of the authors (A. P.) is cordially indebted to the Physics Dept. at the EMU of Famagusta for a visiting position at this Department owing to what completed this work was finished.
[1] U. Taneri, K. Huseyin, P. Yu, Dynamics and Stability of Systems, 10, 145 (1995).

[2] J. M. Yuan, G. C. Lie, Molecular bistability and chaos. Modern Nonlinear Optics. Part 3, (John Wiley \& Sons.Publ., 1994).

[3] A. K. Prykarpatsky, I. V. Mykytyuk, Algebraic integrability of nonlinear dynamical systems on manifolds: classical and quantum aspects (Kluwer Academic Publ., DordrechtBoston-London, the Netherlands, 1998).

[4] V. I. Yukalov, A. S. Shumovsky, Lectures on Phase Transitions (World Scientific Publ., Singapore, 1990).

[5] D. F. Walls, G. J. Milburn, Quantum Optics (Springer-
Verlag, 1994).

[6] N. N. Bogoliubov, N. N. Bogoliubov (Jr), Introduction to Quantum Statistical Mechanics, (World Scientific Publ., 1984).

[7] A. Abrikosov, L. Gor'kov, I. Dzyaloshinski, Methods of quantum field theory in statistical physics (Dover Public. Inc., New York, 1975).

[8] N. N. Bogoliubov, D. V. Shirkov, Introduction to quantum field theory (Prentice Hall, NJ, 1970).

[9] N. N. Bogoliubov, Izv. Akad. Nauk SSSR, Ser. Fiz. 11, 77 (1947).

\title{
РІВНОВАЖНІ РАДІЯЩІЙНІ СТАНИ ОДНІЕЇ НЕЛІНІЙНОЇ КВАНТОВО-ОПТИЧНОЇ СИСТЕМИ
}

\author{
М. М. Боголюбов ${ }^{1}$, У. Танері ${ }^{2}$, А. К. Прикарпатський ${ }^{3}$ \\ ${ }^{1}$ Математичний інститут імені В. А. Стеклова РАН, Відділ статистичной механіки \\ Москба, Російсъка федерачіл \\ ${ }^{2}$ Східно-Середземноморсъкий університет, факультет математики, Фамагуста, Кіпр \\ ${ }^{3}$ Гірничо-металурайна академіл, факультет прикладной математики, Краків, зоо59, Польща \\ та Східно-Середземноморсъкий університет, факультет фізики, Фамагуста, Кіпр.
}

Багато сучасних електронних приладів функпіонують за допомогою оптичних сигналів, генерованих завдяки взаємодії робочого середовища типу кристала зі зовнішнім лазерним випромінюванням. Оскільки в більшості пих приладів взаємодія $є$ нелінійною, а процеси, шо іх стабілізують, квантові, то важлива розробка відпові,дних підходів до вивчення іхніх рівноважних радіяпійних станів стосовно до зовнішніх умов, накладених на систему. Нам вдалося знайти замкнений набір співвідношень на зовнішні параметри системи кристал-світло, що приводить до квазірівноважних сигналу та інтенсивности станів, випромінюваних усередину кристала. Це $\mathbf{i} \epsilon$ основною метою запропонованої праці, у якій розвинуто сучасні квантові теоретико-польові методи аналізу радіяційних станів стосовно однієі нелінійної квантово-оптичної системи, що має важливі застосування в електроніці. 